\title{
El desarrollo profesional online de profesores de matemáticas en activo: una unidad de aprendizaje sobre la enseñanza de la modelización matemática
}

\section{The Online Professional Development on in-Service Mathematics Teachers: A Course about the Teaching of Mathematical Modelling}

\section{Avenilde Romo-Vázquez*}

(iD) https://orcid.org/0000-0002-1364-5997

$$
\text { Berta Barquero** }
$$

(iD) http://orcid.org/0000-0001-7228-6210

\section{Marianna Bosch ${ }^{* * *}$}

(iD) https://orcid.org/0000-0001-9756-116X

Tipo de Artículo: Reportes de investigación y ensayos inéditos

Doi: 10.17533/udea.unipluri.19.2.09

Cómo citar este artículo:

Romo-Vázquez, A., Barquero, B., y Bosch, M. (2019). El desarrollo profesional online de profesores de matemáticas en activo: una unidad de aprendizaje sobre la enseñanza de la modelización matemática.

Uni-pluriversidad, 19(2), 161-183. doi: 10.17533/udea.unipluri.19.2.09

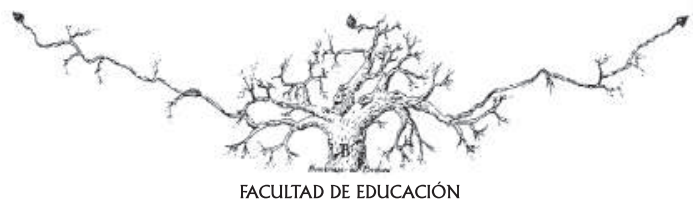

Recibido: 2019-10-23 • Aprobado: 2019-12-14

* CICATA, Instituto Politécnico Nacional, Mexico

E-mail: aromov@ipn.mx

** Faculty of Education, University of Barcelona, Barcelona, Spain

E-mail: bbarquero@ub.edu

*** IQS School of Management, Ramon Llull University, Barcelona, Spain.

E-mail: marianna.bosch@iqs.url.edu 


\section{Resumen}

El desarrollo profesional online del profesorado permite articular la difusión de nuevas propuestas basadas en la investigación didáctica con la experiencia del docente y sus dificultades para integrarlas en el aula. Presentamos una investigación sobre el diseño e implementación de Unidades de Aprendizaje online y a distancia para profesores de matemáticas en activo basada en una adaptación de la metodología de los Recorridos de Estudio e Investigación para la Formación del Profesorado (REI-FP) propuesta por Ruiz-Olarría (2015). De acuerdo con esta metodología, la Unidad de Aprendizaje (en adelante, UA) pretende abordar, junto con los profesores participantes, los obstáculos institucionales que plantea la enseñanza de la modelización matemática en relación con la enseñanza por transmisión de conocimientos preestablecidos: rigidez del currículo, gestión del tiempo, escasez de dispositivos de evaluación adaptados, control del uso de las TIC, multidisciplinariedad, entre otros.

La investigación se basa en un estudio de caso formado por cuatro ediciones sucesivas de una UA para profesores de matemáticas latinoamericanos en activo que se realizó en el Centro de Investigación en Ciencia Aplicada y Tecnología Avanzada de México. La UA se centra en una actividad de modelización sobre previsiones de ventas que adapta la metodología de los REI-FP al caso online y a distancia. Después de describir el contenido de la UA, se realiza un análisis clínico exploratorio del proceso de formación implementado, usando como material empírico las producciones de un grupo de profesores. Los resultados muestran la viabilidad de la adaptación metodológica, poniéndose de manifiesto que los dispositivos de formación implementados facilitan la emergencia e identificación de restricciones institucionales generalmente ocultas a los docentes en su quehacer cotidiano. También muestran limitaciones del proceso de formación que requieren mayor explotación de las herramientas multimedia, así como la necesidad de extender el proceso de formación impartido más allá de la estricta temporalidad de la UA.

Palabras clave: Educación continua del profesorado, (Unidad de Aprendizaje) UA online, didáctica de las matemáticas, modelización matemática, recorridos de estudio e investigación.

\section{Abstract}

Online professional development of teachers enables to connect the dissemination of new proposals based on educational research to teachers' experience and their difficulties when they are trying to integrate those proposals into the classroom. We present a research 
focused on the design and implementation of online and distance learning courses for in-service mathematics teachers based on an adaptation of the methodology of "Study and Research Paths for Teacher Education" (SRP-TE) by Ruiz-Olarría (2015). According to this methodology, the course aims at addressing, together with the participating teachers, the institutional obstacles that appear when mathematical modelling is taught. These obstacles are related to traditional pedagogical practices based on the transmission of pre-established knowledge: curricular rigidity, time management, lack of adapted assessment devices, use of ICT, multidisciplinary aspects, etc.

The research is based on a case study consisting of four successive editions of a course for Latin American mathematics teachers held by the Centro de Investigación en Ciencia Aplicada y Tecnología Avanzada in Mexico. The course focuses on a modelling activity about sales forecasts and it adapts the SRP-TE methodology to online and distance learning education. After describing the course organization, we carry out an exploratory clinical analysis of the implemented educational process considering the evidence provided by the productions of a group of teachers. The results show the viability of the methodological adaptation, showing how the educational devices facilitate the emergence and identification of institutional constraints that generally remain hidden in the teacher's daily work. The results also highlight limitations of the course that require greater exploitation of multimedia tools, as well as the need to extend the educational process beyond its strict length.

Keywords: In-service Teacher Education, Online Courses, Mathematics Education, Mathematical Modelling, Study and Research Path. 


\section{INTRODUCCIÓN:}

\section{LA FORMACIÓN DE PROFESORES ONLINE, POSIBILIDADES Y LÍMITES}

La educación matemática de calidad ha sido planteada como una necesidad de la sociedad actual, cuya satisfacción está estrechamente relacionada con el desempeño del profesor, y por ende, con su formación inicial y continuada. Artigue (2016) caracteriza esta última de la manera siguiente:

El desarrollo profesional de los profesores debe estar sostenido por la organización de una formación continua específica, permitiendo a estos últimos estar en contacto con la evolución de su disciplina, de apoyarse para su trabajo en los resultados de la investigación didáctica, de sacar provecho de la evolución tecnológica, de adaptar su enseñanza tanto en sus contenidos como en sus prácticas a la evolución de las prácticas y las demandas sociales. (p. 36, traducción propia)

Esto plantea un reto: el profesor de matemáticas debe conocer elementos -marcos teóricos, marcos metodológicos, herramientas, postulados, resultados- de la investigación en didáctica de las matemáticas, sin alejarse de su aula. Para afrontarlo, la enseñanza a distancia permite al profesorado combinar su formación continua con la práctica docente $\mathrm{y}$, por lo tanto, experimentar de forma paralela los contenidos de la formación con la realidad del aula. Lo antes expuesto conduce a analizar las potencialidades de la modalidad online y a distancia. Para ello, se consideran diferentes trabajos de investigación desarrollados en el dominio de la educación y la Didáctica de las Matemáticas (DM). Barberá y Fuentes (2012) muestran las posibilidades de esta modalidad al ilustrar el diseño de un curso de for- mación en inteligencia emocional aplicada al aula, dirigido al profesorado de Educación Primaria y Secundaria. Los autores enfatizan que los participantes deben tener conocimientos sobre las TIC y el diseño del curso debe ser acorde al uso de herramientas multimedia como son videos, foros, Wikis, cuestionarios online de corrección entre otros. Desde nuestro punto de vista, supone una concepción distinta de los contenidos y también una forma diferente de "construirlos" a través de la tecnología informática. Costa (2009) apunta la importancia de las herramientas de segunda generación (web 2.0) para ayudar a los estudiantes a desarrollar su propia trayectoria de aprendizaje, autónomamente y en comunidad, en entornos más dinámicos. La utilidad de estos entornos resulta particularmente importante en el caso de la formación del profesorado ya que, en general, la experiencia vital de los profesores constituye un elemento insustituible en su práctica docente:

Teachers, as 'actors of change', specialized in preparing the younger generations to the new job market should see in informal forms of learning, mediated by web 2.0 environments, a potential alternative to standard, traditional teaching and teacher training. (Costa, 2009, p. 3)

Los entornos virtuales potencian, además de la dimensión colectiva del desarrollo profesional docente, la generación de una Comunidad Local de Práctica en el sentido de Winbourne y Watson (1998), que pueden perdurar más allá del propio proceso de formación. En el marco del enfoque socio-cultural del aprendizaje, el informe de 
Wideman (2010) recoge los principales resultados expuestos en la literatura sobre el uso de comunidades de aprendizaje online para el desarrollo profesional docente y la importancia para el aprendizaje profesional de los docentes de participar en prácticas reflexivas, de compartir sus experiencias con otros colegas igualmente involucrados. En este sentido, Scott (2010) aporta evidencia empírica sobre la efectividad de los programas de formación del profesorado, indicando seis principios básicos que también recogen otros autores como Castañeda y Adell (2011):

1. Take a problem-solving orientation;

2. Incorporate opportunities for teachers to work together, and with experts;

3. Facilitate exposure to innovations in knowledge, teaching practice, and supporting technologies;

4. Enable teachers to try out in whole new teaching strategies and skills;

5. Promote the creation and sharing of resources;

6. Enable ongoing and purposeful reflections and discussion.

El cómo respetar estos principios en los programas de formación online y a distancia requiere un diseño específico que tenga en cuenta, particularmente, la disciplina objeto de enseñanza. En el caso de la formación del profesorado de matemáticas, existen algunas experiencias pioneras a principios del siglo XXI (por ejemplo, Garuti, 2004; Ponte y Santos, 2004; Borba y Zulatto, 2006; McGraw, Lynch, Koc, Budak y Brown, 2007; Gueudet, Sacristán, Soury-Lavergne y Trouche, 2012; Galleguillos y Borba, 2017). Des- de entonces se desarrollan diferentes líneas de investigación que se focalizan en la integración de las tecnologías en la enseñanza de esta disciplina, en las diferentes formas de interacción que pueden generarse en la modalidad online (mail, chat, foro, videoconferencia, etc.); en temas específicos, modelización matemática (Barquero, Bosch y Romo, 2018; Orey y Rosa, 2018; Parra-Zapata, et al., 2018), el diseño de actividades didácticas (Romo y Covián, 2018), el lenguaje matemático como objeto de estudio (Romo y Hache, 2019) y la creación de comunidades de aprendizaje (Romo-Vázquez y Gómez-Blancarte, 2018). Muchas están destinadas a la formación inicial del profesorado o combinando la formación presencial con actividades online (Llinares y Valls, 2010). En general, se preconizan actividades que se alejen del modelo transmisivo (subir material en diferentes soportes y solicitar comentarios por parte de los estudiantes) para recurrir a nuevos dispositivos que permitan generar un rápido feedback entre los participantes, que faciliten la comunicación y la construcción colectiva de nuevas ideas y propuestas educativas, y que promuevan una interacción ágil entre profesores en formación y formadores.

En un marco más amplio que abarca los programas de desarrollo profesional de profesores en activo (presenciales y a distancia), una recensión de la investigación en el área (Goldsmith, Doerr y Lewis, 2014) señala la falta de una descripción sistemática y global de la naturaleza del enfoque que se adapte en los programas, incluyendo las actividades concretas que se proponen a los profesores así como el tiempo dedicado a cada una de ellas. Es necesario, como lo indican estos autores, "to know more about the specific content of activities and, for facilitated professional learning experiences, 
the nature of facilitation" (p. 22). Es por ello que en este artículo se analiza una UA, mostrando las actividades que la conforman y el marco institucional constituido para que los profesores experimenten, analicen, adapten e implementen un material didáctico en el aula, enmarcado en los recorridos de estudio y de investigación para la formación del profesorado, como se detalla a continuación.

\section{LOS RECORRIDOS DE ESTUDIO E INVESTIGACIÓN PARA LA FORMACIÓN DEL PROFESORADO}

En sintonía con el paradigma socio-cultural de la formación del profesorado, investigaciones desarrolladas en el marco de la Teoría Antropológica de lo Didáctico (TAD) plantean la necesidad de basar el desarrollo profesional del profesorado de secundaria en cuestiones problemáticas que surgen durante el ejercicio de la profesión (Cirade, 2006; Bosch y Gascón, 2009; Barquero, Florensa y Ruiz-Olarría, 2019). Se consigue que el proceso de formación permita vincular los nuevos conocimientos fruto de la investigación educativa con la realidad del aula y presentarlos como herramientas para analizar y resolver problemas, más que como un conjunto de técnicas y saberes más o menos dogmáticos (Chevallard, 2013). Al mismo tiempo, los conocimientos didácticos se pueden utilizar para cuestionar tanto los contenidos curriculares como las formas de enseñanza, permitiendo a los profesores en formación formular nuevas cuestiones problemáticas que entorpecen implícitamente el quehacer docente, actuando de forma transparente para todos los actores del proceso educativo.

Estas asunciones se materializan en una metodología designada como "Recorridos de Estudio e Investigación para la Formación del Profesorado" (REI-FP) experimentada inicialmente por Sierra (2006) en el caso de la formación inicial de maestros y desarrollada por Ruiz-Olarría (2015) para la formación inicial de profesores de matemá- ticas de secundaria. En esta metodología, el proceso de formación parte de una cuestión problemática para la profesión docente que se aborda en cuatro etapas generales: vivir, como estudiante, una propuesta innovadora de actividad matemática; analizarla y adaptarla para su implementación en el aula; experimentarla con alumnos de secundaria; identificar las restricciones institucionales que la experimentación ha puesto en evidencia y refinar o rediseñar la actividad.

Esta metodología de formación ha sido adaptada a la modalidad en línea y a distancia considerando herramientas multimedia como foros, videos, plataforma Moodle, así como las condiciones del trabajo asíncrono y el hecho de que los participantes son profesores en servicio de educación secundaria o universitaria (Barquero, Bosch y Romo, 2018). Particularmente, la condición de profesores en servicio permite que puedan realizar experimentaciones en sus clases o con pequeños grupos de estudiantes voluntarios.

La investigación que aquí se presenta tiene un doble propósito:

1. Analizar los efectos de la implementación de una UA y la forma en que ésta posibilita a los profesores analizar, adaptar, validar y desarrollar una propuesta didáctica sobre la enseñanza de la modelización matemática. 
2. Observar en qué medida los profesores logran, durante la UA y gracias a las herramientas propuestas en ella, identificar las principales restricciones institucionales que dificultan la integración de la modelización matemática como actividad normalizada en el aula.

\section{Contexto experimental y metodología}

El programa de Matemática Educativa del Centro de Investigación en Ciencia Aplicada y Tecnología Avanzada del Instituto Politécnico Nacional de México ofrece desde el año 2000 una maestría y un doctorado, en línea y a distancia, para profesores de matemáticas en servicio que otorga una gran importancia a la integración explícita de nuevos conocimientos en didáctica de las matemáticas a la práctica docente efectiva (Gómez-Blancarte, Romo-Vázquez, Miranda, Aguilar, Lezama y Castañeda, 2019). La maestría se organiza en cuatro semestres en los que se ofrecen 12 UA's que son impartidos en los primeros tres semestres, con el objetivo de mostrar una perspectiva general de la DM, de sus herramientas teóricas y metodológicas, así como la forma en que éstas pueden utilizarse para gestionar, regular y evolucionar la práctica docente. El cuarto semestre está dedicado íntegramente al desarrollo de un pequeño trabajo de investigación que surja en relación con algún problema de la propia práctica docente.

La metodología de investigación seguida se basa en un análisis clínico (Pedinielly y Fernandez, 2015) y exploratorio de las producciones de un grupo de profesores participantes en la UA titulada "Procesos de Institucionalización de la Matemática Escolar" (en adelante, PIME), cuyas características se presentan a continuación así como los insumos considerados para su análisis.

\section{Características de la UA PIME enfocada en la enseñanza de la modelización matemática.}

La UA PIME se enfocó en la enseñanza de la modelización matemática y de las dificultades o limitaciones de índole institucional (contexto escolar, condiciones curriculares, etc.) que emergen o podrían emerger en su inclusión en el aula. Su diseño se basó en varias cuestiones de la profesión: ¿Cómo analizar, adaptar, desarrollar e integrar a la práctica docente un proceso didáctico relacionado con la modelización matemática? ¿Cómo difundir entre pares e institucionalizar a largo plazo un proceso didáctico basado en la modelización y que se considera pertinente para determinado nivel de formación escolar? ¿Qué dificultades deben superarse? ¿Qué herramientas didácticas lo posibilitan? ¿Qué nuevas cuestiones surgen y en qué instancias pueden abordarse? Su duración fue de cuatro semanas y se desarrolló en un espacio de la plataforma Moodle en el que eran visibles el nombre de los formadores responsables, un video de presentación, un documento en el que se detallaban los objetivos, las cuatro actividades a desarrollar, el calendario y la forma de evaluación. La actividad de los profesores participantes se desarrolló principalmente en los foros asociados a las cuatro actividades, lo que les permitió generar los entregables solicitados, mediante documentos escritos. La actividad de los formadores se caracterizó por intervenciones realizadas mediante videos (retroalimentaciones generales), foros (retroalimentaciones o moderación de debate en pequeño grupo), mail (individual o grupal) y la puesta a disposición de documentos de soporte (artículos, material didáctico, pautas, etc.) para el desarrollo de las actividades. 


\section{Insumos para el análisis}

La UA se impartió durante el primer semestre de cuatro años académicos (2013/14, 2014/15, 2015/16 y 2017/18). Para el análisis, se eligió la segunda edición 2014/15, ya que constituyó un caso representativo y tuvo dos características distintivas: un diseño afinado de la UA -basado en el análisis de la primera edición- $\mathrm{y}$ un uso privilegiado de los foros en el desarrollo de las actividades. La riqueza de interacciones entre profesores en esta edición es única, ya que en las ediciones más recientes los profesores utilizan herramientas como el Whatsapp y el Skype, lo que impide a los formadores observar al desarrollo completo de las actividades. Los insumos considerados para el análisis fueron las intervenciones en los foros, los reportes, los documentos escritos y los resultados derivados de las encuestas de evaluación.

\section{Análisis de la UA enfocada en la enseñanza de la modelización matemática-PIME-}

La UA PIME estuvo conformada por cuatro grandes actividades que situaron a los profesores en diferentes roles: matemático o aprendiz matemático, docente y didacta. La primera actividad consistía en desarrollar el REI "Previsión de ventas para Desigual" y, a partir de un conjunto de datos, hacer una previsión de ventas a corto y largo plazo. La segunda actividad consistía en realizar una guía didáctica para que un colega implementara el REI "Previsión de ventas para Desigual" en el aula. Lo que requería una primera reflexión didáctica sobre el REI experimentado en la primera actividad. En la tercera actividad se solicitaba adaptar el REI "Previsión de ventas para Desigual" para ser implementado con un grupo de estudiantes de secundaria. Esta actividad constituye el corazón de la UA pues los profesores deben poner en juego la experiencia vivida en la primera actividad así como sus conocimientos profesionales para realizar la adaptación, haciendo visibles las restricciones institucionales que imperan sobre la enseñanza. $Y$ finalmente, en la cuarta actividad se solicitaba hacer un análisis de la implementación hecha en la tercera actividad, que incluía un análisis matemático y didáctico del REI adaptado "Previsión de ventas para Desigual". Para realizar esta actividad, diferentes herramientas teóricas y metodológicas fueron propuestas. A continuación, se presentan estas cuatro actividades en detalle, acompañadas del análisis de producciones realizadas por los profesores, que ilustran la forma en que analizaron, adaptaron, validaron y desarrollaron una propuesta didáctica -REI "Previsión de venta para Desigual"sobre la enseñanza de la modelización matemática.

\section{Actividad 1 \\ Previsión de ventas para Desigual}

La actividad de 'Previsión de ventas para Desigual' constituye un REI diseñado por Serrano (2012), en el que se pide realizar una previsión a corto y largo plazo de las ventas que realizará la empresa Desigual. Se trata de una actividad abierta, en la que cada equipo, compuesto por tres profesores, se organiza libremente para estudiar dos de las once variables (ventas de camisetas, ingresos de la tienda, etc.), asumiendo un rol de matemático o matemático aprendiz.

La consigna fue presentada de la siguiente manera: La Consultora matemática "Pi \& SA" solicita a su departamento de consultores junior que respondan al encargo de la empresa DESIGUAL bajo la responsabilidad de los consultores senior 
-nombres de formadores-. Todos los participantes, en el rol de aprendices matemáticos o matemáticos, se convertirán en una consultoría matemática y trabajarán en grupos de consultores (de 3 o de 4 personas). Todos los grupos abordarán la misma cues- tión aunque cada subgrupo tendrá asignadas un par de variables distintas.

Para ilustrar el tipo de variables y los datos asociados se muestran tres de las 11 variables:

1) Número de tiendas propias abiertas a nivel nacional en los últimos trimestres:

\begin{tabular}{|c|c|c|c|c|c|c|c|c|c|c|}
\hline Trimestre & T1 2008 & T2 2008 & T3 2008 & T4 2008 & T1 2009 & T2 2009 & T3 2009 & T 4 2009 & T 12010 & T 2 2010 \\
\hline Tiendas abiertas & 15 & 19 & 12 & 22 & 20 & 25 & 24 & 35 & 45 & 55 \\
\hline
\end{tabular}

2) Número de tiendas propias abiertas fuera de España en los últimos trimestres:

\begin{tabular}{|c|c|c|c|c|c|c|c|c|c|c|}
\hline Trimestre & T1 2008 & T2 2008 & T3 2008 & T4 2008 & T1 2009 & T2 2009 & T3 2009 & T 4 2009 & T 1 2010 & T 2 2010 \\
\hline Tiendas abiertas & 2 & 4 & 3 & 4 & 6 & 7 & 8 & 15 & 32 & 44 \\
\hline
\end{tabular}

3) Evolución de las ventas anuales contabilizadas en millones de euros:

\begin{tabular}{|c|c|c|c|c|c|c|}
\hline Año & 2004 & 2005 & 2006 & 2007 & 2008 & 2009 \\
\hline Millones de curos & 19 & 30 & 49 & 86 & 162 & 230 \\
\hline
\end{tabular}

Figura 1. Variables y datos asociados para realizar la previsión de ventas a corto y largo plazo

Esta actividad se realiza en tres fases (Figura 2). En la fase 1 se presenta el documento "Previsión de ventas para Desigual" con los datos sobre las variables a estudiar y se habilita un foro para que el equipo de profesores realice el trabajo matemático asociado a la previsión: ajuste de datos, búsqueda de modelos matemáticos, criterios de selección del mejor modelo, formas de contraste y validación, etc. Esta actividad se reporta en un "informe intermedio" conteniendo una primera previsión, su justificación y nuevas cuestiones. En la fase 2 se contrastan los informes intermedios producidos por dos equipos, se genera una reflexión conjunta sobre el trabajo y su continuación (Figura 3 -"Foro 2"-). En la fase 3 se presenta un informe final del estudio de las variables dirigido a la empresa Desigual, así como una descripción del proceso vivido dirigido a la Consultora -cuyos representantes son los formadores-. En este mismo informe se pide incluir nuevas cuestiones que resultan del estudio elaborado y que podrían ser útiles para continuarlo, lo que muestra que la respuesta no es "una respuesta final" sino una respuesta válida y consensuada, pero siempre mejorable.

\section{Actividad 1}

Fase 1

Foro 1. Equipo 1

Foro 1. Equipo 2

Foro 1. Equipo 3

Foro 1. Equipo 4

Foro 1. Equipo 5

Primer informe
Fase 2

Fase 3

Figura 2. Fases de la Actividad 1 en la plataforma de la UA

Informe final 
Para ilustrar el desarrollo de la actividad 1, se eligió analizar el informe final producido por el equipo $\mathrm{A}$, formado por dos profesores mexicanos y uno uruguayo. Este informe que se centra en el estudio de las previsiones de las variables 5 y 6 (Figura 3 ) se basa tanto en el informe intermedio como en la discusión generada en la fase 2, con el equipo B.

1) Evolución de los ingresos semanales ( $€$ ) desde el 15 de febrero de 2010 en la tienda de las Ramblas 136 (Barcelona):

\begin{tabular}{|c|c|c|c|c|c|c|c|c|c|c|c|}
\hline Semana & S1 & S2 & S3 & S4 & S5 & S6 & S7 & S8 & S9 & S10 & S11 \\
\hline $\begin{array}{c}\text { Ingresos } \\
\text { semanales }\end{array}$ & 52500 & 25250 & 25000 & 26750 & 28790 & 31300 & 35000 & 34990 & 41000 & 43550 & 45570 \\
\hline
\end{tabular}

2) Evolución de los ingresos semanales $(€)$ desde el 1 de marzo de 2010 en la tienda de la Rambla de Catalunya 140 (Barcelona):

\begin{tabular}{|c|c|c|c|c|c|c|c|c|c|c|}
\hline Semana & S1 & S2 & S3 & S4 & S5 & S6 & S7 & S8 & S9 & \$10 \\
\hline $\begin{array}{c}\text { Ingresos } \\
\text { semanales }\end{array}$ & 25000 & 25450 & 26750 & 28800 & 31300 & 35050 & 34995 & 40975 & 43700 & 45973 \\
\hline
\end{tabular}

Figura 3. Variables 5 y 6

Este equipo muestra en el informe final una exploración sobre distintos modelos matemáticos, funciones lineales, polinómicas, exponenciales, logarítmicas y potenciales,
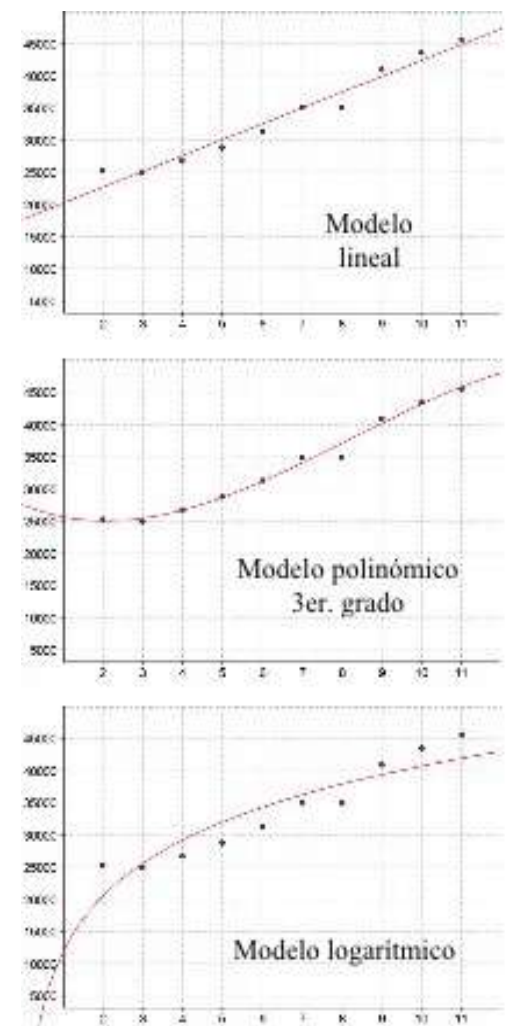

como modelos de ajuste para las variables 5 y 6. En la figura 4 se ilustran sus diferentes propuestas en relación con la variable 5 :
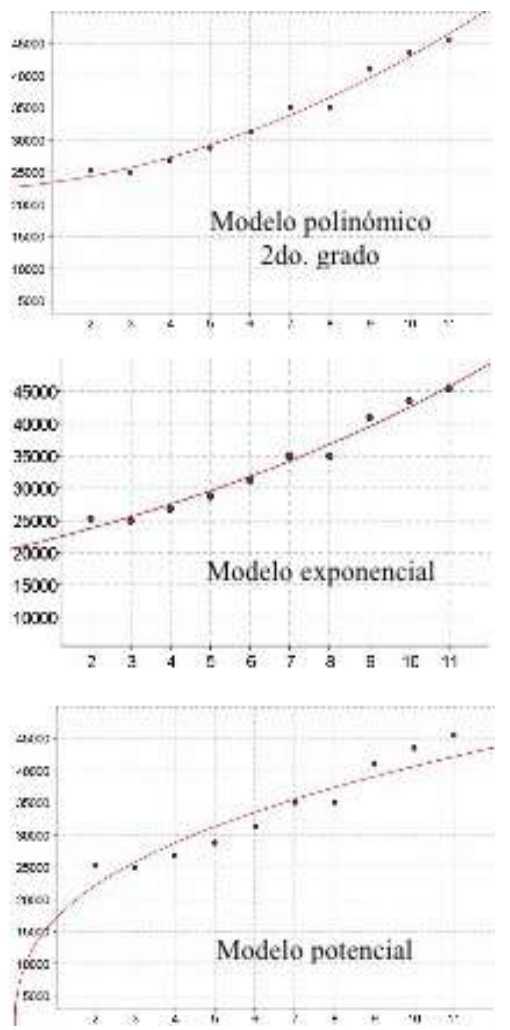

Figura 4. Modelos de ajuste propuestos por el equipo A

Un primer análisis de sus diferentes propuestas los lleva a eliminar uno de los datos reales y mejorar tanto el ajuste como la previsión. Posteriormente, aparecen dos 
nuevas cuestiones que guían el desarrollo del estudio: ¿Qué criterios se deben emplear para elegir el modelo más adecuado? ¿Qué debe entenderse por largo y corto plazo? Para abordar la primera cuestión eligen el uso del coeficiente de determinación $\mathrm{R}^{2}$ para cada uno de los modelos analizados (Tabla 1). Este primer análisis se complementa por

Tabla 1.

Valores del coeficiente $R^{2}$

\begin{tabular}{|c|c|c|}
\hline Modelo & $\begin{array}{c}\text { Variable } \\
\mathbf{5}\end{array}$ & $\begin{array}{c}\text { Variable } \\
\mathbf{6}\end{array}$ \\
\hline Lineal & 0,962 & 0,963 \\
\hline Polinómico 2do. grado & 0,983 & 0,986 \\
\hline Polinómico 3er. grado & 0,987 & 0,988 \\
\hline Exponencial & 0,979 & 0,981 \\
\hline Logaritmico & 0,842 & 0,784 \\
\hline Potencial & 0,894 & 0,843 \\
\hline
\end{tabular}

En cuanto a la segunda cuestión, en su primer informe habían elegido para el corto y largo plazo 3 y 12 meses respectivamente, mientras que en el final eligen 6 y 36 meses. Este cambio, señalan, se originó en el intercambio con el equipo B (fase 2 de la Actividad 1), y considerando la frecuencia con que se ajustan los salarios de los consumidores, el gran volumen de ventas de la empresa Desigual, así como sus planes de expansión nacional e internacional. Todo lo anterior muestra la naturaleza de la actividad de modelización llevada a cabo por los profesores, donde se combina elementos matemáticos con conocimientos propios al contexto: errores en los datos, posibilidades de quiebra, situación económica de la empresa. En cuanto a los recursos, indican el programa computacional GeoGebra que les permitió tomar decisiones sobre el modelo adecuado, y una breve bibliografía de estadística para investigar sobre la regresión.

Los profesores reconocen que en esta actividad hay una diversidad de conceptos otro, apoyado en la coherencia que puedan tener las previsiones dadas por los modelos en el contexto real de ventas que se consideró. Por ejemplo, señalan que los modelos polinómicos de grado 3 o más pronostican una quiebra prematura de la empresa que no corresponde con la expansión actual de Desigual (Figura 5).

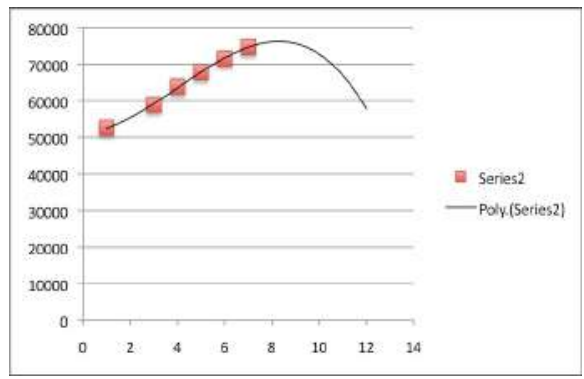

Figura 5. Modelo cúbico (semana 14)

y "estrategias" matemáticas implicadas que asocian a la estadística, familias de funciones, análisis de modelos matemáticos como potenciales y logarítmicos, coeficientes de correlación y determinación. Mencionan que "implícitamente" aparecieron conceptos como los de varianza y error estadístico. Esto hace suponer que el trabajo es mucho mayor al expuesto en los dos informes y que su decisión de mostrar o no mostrar puede estar asociada al avance de uso de estos conceptos. Por ejemplo, indican que una dificultad consiste en equilibrar lo matemático y lo contextual: "Nos costó desprendernos del coeficiente de determinación y asumir que la interpretación de los modelos a la luz de la realidad económica de la empresa era tan importante como el aspecto técnico matemático". Este punto de vista se refuerza cuando en las nuevas cuestiones señalan: "Entendemos que son necesarios más datos para realizar una previsión más confiable. Muchos modelos con buen coeficiente de determinación proyectan un crecimiento 
cuya viabilidad depende de la capacidad real de expansión de las tiendas consideradas" [Equipo A, informe final]. Se puede notar cómo en esta actividad vivida por el equipo A se realizan tareas de investigación de diferentes niveles (por ejemplo, exploratorias, de análisis, de conjetura, de interpretación y de validación). Las respuestas no son presentadas como únicas ni definitivas sino que muestran lo que aportan y lo que debe desarrollarse.

\section{Actividad 2}

\section{Producción de una guía didáctica.}

La Actividad 2 consiste en el desarrollo de una guía didáctica basada en un análisis didáctico espontáneo. Es decir, la forma de presentar la consigna y el tiempo dado para desarrollarla obliga a los profesores a situarse en el rol de docentes -su rol habitual-y de reflexionar sobre el cómo el REI experimentado en la primera actividad puede implementarse en el aula. Las preguntas que pueden estar en la base de este análisis son las siguientes: ¿Qué se estaría enseñando con el REI "Previsión de ventas para Desigual", funciones, elementos de estadística? ¿Qué conocimientos requieren los estudiantes para desarrollar el REI? ¿Cuál sería el rol del profesor? ¿Un rol tradicional o un rol parecido al seguido por los formadores (pocas indicaciones, cuestionando sobre criterios, animando a investigar, etc.)? ¿Los estu- diantes podrían desarrollar el REI de manera autónoma? ¿La actividad puede ser abierta o la incertidumbre de no saber qué se pide exactamente limitaría a los estudiantes en el desarrollo del REI?, etc. La Actividad 2 se desarrolla en tres fases:

Fase 1. Realizar una guía didáctica de manera individual para que un colega implemente con un grupo de estudiantes la actividad "Previsión de ventas para Desigual".

Fase 2. Analizar y discutir en un foro las guías didácticas producidas individualmente por cada miembro del equipo, para ello se propone considerar diferentes elementos: organización de la clase (gran grupo, grupos pequeños), actividad del profesor, actividad de los estudiantes, el tipo de problema propuesto (abierto, de investigación, tarea matemática tradicional, de respuesta inmediata), conocimientos con los que cuentan los estudiantes, conocimientos que van a construirse, objetivos de la actividad, tema del programa que se aborda, discurso del profesor, etc.

Fase 3. Generar una guía didáctica común. El objetivo de esta actividad es situar a los profesores en un nivel de análisis didáctico de la actividad "Previsión de ventas para Desigual", para estudiar su viabilidad en las aulas de secundaria, sus potencialidades y limitaciones así como la manera de adaptarla a las restricciones propias de la educación secundaria (conocimientos previos, tiempos y organización de la clase).

Actividad 2

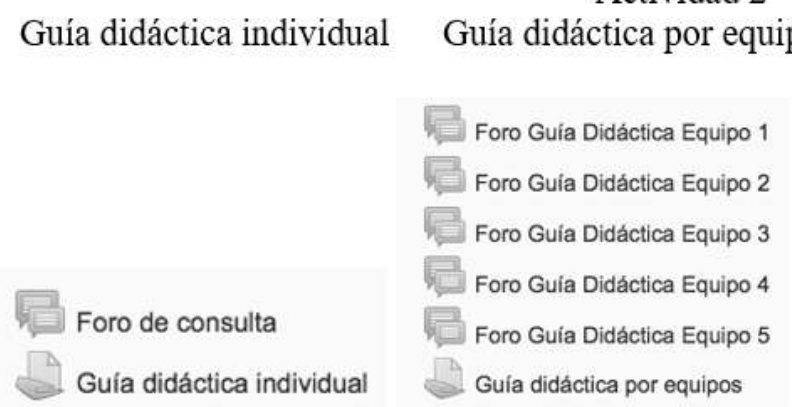

Video de retroalimentación de los formadores

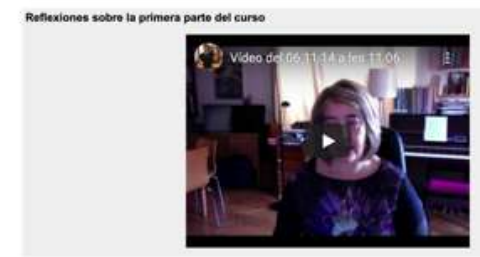

Figura 6. Actividad 2 en la plataforma 


\section{Guía didáctica del equipo $A$.}

La guía didáctica producida por el equipo A tiene cuatro partes: en la primera se presentan características de la actividad didáctica de modelización matemática, modelo matemático o tema abordado, conocimientos previos, objetivos y organización de la clase; en la segunda se indica la consigna de la actividad y su fragmentación en tareas; en la tercera se precisa la actividad esperada por el profesor y los estudiantes; y en la cuarta, nuevas cuestiones. Estas cuatro partes, como se muestra a continuación, se basan tanto en la experiencia docente de los profesores como en lo vivido en la Actividad 1. El tema propuesto es "Funciones polinómicas y modelización matemática" $\mathrm{y}$ se solicita el recurso al software matemático educativo GeoGebra. En los objetivos de la actividad se señala: resolver un problema abierto fomentando la formulación y validación de conjeturas e incentivar el trabajo colaborativo y la discusión entre pares. La organización de clase consta de tres etapas, para desarrollar por equipos de estudiantes en un tiempo de 90 a 120 minutos:

1. Presentación de la actividad a los estudiantes y exploración de GeoGebra;

2. Realización de la actividad;

3. Puesta en común e institucionalización de los conocimientos involucrados.

La actividad propuesta a los estudiantes, como se indica en la parte 2 de la guía didáctica, está compuesta de tres grandes tareas, conservando la "Previsión de ventas para Desigual" a corto plazo (próximas semanas) de la variable 6 :

\section{6) Evolución de los ingresos semanales $(€)$ desde el 1 de marzo de 2010 en la tienda de la Rambla de Catalunya 140 (Barcelona):

\begin{tabular}{|c|c|c|c|c|c|c|c|c|c|c|}
\hline Semana & S1 & S2 & S3 & S4 & S5 & S6 & S7 & S8 & S9 & S10 \\
\hline Ingresos semanales & 25000 & 25450 & 26750 & 28800 & 31300 & 35050 & 34995 & 40975 & 43700 & 45973 \\
\hline
\end{tabular}

Figura 7. Variable 6 de la Actividad 1 "Previsión de ventas para Desigual"

La situación de partida se describe como "Los directivos de la empresa están interesados en saber qué ingresos podrán obtener en dicha sucursal, al cabo de las próximas semanas." Se propone una primera tarea que consiste en graficar los datos de la tabla en GeoGebra, insertando dos deslizadores $a$ y $b$ y representando la familia de funciones afines $y=a x+b$. A continuación, se pide a los alumnos que elijan la mejor recta que ajusta los datos y justifiquen su elección. Finalmente, los estudiantes deben hacer una previsión de los ingresos de la última semana del año (semana 17) e indicar cómo la obtienen.
El equipo A señala que esta tarea se propone para que los estudiantes analicen el modelo lineal y el uso de los deslizadores, que aportan dinamismo a la actividad. En el apartado 3 de la guía se reconoce que esta primera tarea es exploratoria y que el profesor que la implemente deberá "aceptar" los argumentos de los estudiantes, incluso si éstos son poco profundos o inexactos.

El equipo propone una tarea complementaria: De ser necesario, el profesor establecerá el rango de valores que podrá tomar cada deslizador y su velocidad de crecimiento, según se detalla en la siguiente tabla: 
Tabla 2

Rango de valores de los deslizadores

\begin{tabular}{|c|c|c|c|}
\hline Parámetro & Valor mínimo & Valor máximo & Incremento \\
\hline$A$ & -3000 & 3000 & 0,1 \\
\hline$B$ & -30000 & 30000 & 0,1 \\
\hline
\end{tabular}

Esta tarea parece proponerse para "ayudar" al profesor a superar dificultades asociadas al uso de GeoGebra, pero también, puede tener como objetivo "cerrar" la tarea $\mathrm{y}$ asegurar que los estudiantes hagan lo que se espera de ellos. Se puede notar que para los profesores resulta complejo aceptar que una actividad abierta puede ser implementada exitosamente por un docente y desarrollada por los estudiantes. Por eso, tienden a cerrar la actividad.

En la segunda tarea se pide exactamente lo mismo que en la precedente, pero considerando la familia de parábolas $y=c x^{2}+d x+e$. De la misma manera que para la tarea 1 , se precisa que se trata de un trabajo "netamente" experimental y se propone una tarea complementaria para hacer variar los parámetros $c, d$ y $e$ con deslizadores de incremento 0.1 , "cerrando" nuevamente la actividad como en el caso anterior.

Para agregar al análisis un modelo cúbico, se dice a los estudiantes que un estudio mostró que la mejor previsión estaba dada por la función cúbica: $y=-20 x^{3}+590 x^{2}-1000 x+24000$, pidiéndoles manifestar su acuerdo o desacuerdo, así como una justificación. Se esperaba que los estudiantes descartaran este modelo al ver que conducía a la quiebra. Para asegurarse de ello, el profesor explica elementos del contexto como son la situación actual de la empresa y sus proyecciones nacional e internacional.

En la tercera tarea de la guía didáctica se pide a los alumnos comparar los modelos polinómicos de primer, segundo y tercer grado para determinar cuál o cuáles resultan más adecuados y justificar la elección. Esta tarea se declara como "clave" ya que "cada equipo deberá ponderar los criterios empleados para elegir el modelo adecuado: aspectos matemáticos vs. viabilidad de las previsiones". Se indica que, si se considera factible, el profesor "podrá sugerir que se calculen las diferencias entre los datos proporcionados por la tabla y los valores de las previsiones para las semanas ya transcurridas, según cada modelo".

Es posible notar que la adaptación de la actividad "Previsión de ventas para Desigual" está hecha para guiar a los estudiantes en el trabajo con modelos polinómicos de primer, segundo y tercer grado. A pesar de que se considere el uso del programa GeoGebra, que permite la obtención de múltiples representaciones gráficas y su comparación, esta propuesta parece estar fuertemente basada en el paradigma dominante de enseñanza, donde el profesor guía y regula la enseñanza, indicando a los alumnos qué herramientas deben utilizar. Resulta complicado para los profesores suponer que las dificultades que ellos superaron en la Actividad 1 de la UA (ambigüedad de la cuestión, búsqueda de información, etc.), también podrían ser superadas por los estudiantes.

\section{Actividad 3}

\section{Implementación de la actividad "Previ- sión de ventas" con estudiantes.}

En la Actividad 3 de la UA cada profesor implementa con estudiantes, su clase o un grupo de voluntarios, la actividad de 
"Previsión de ventas para Desigual" propuesta en la guía didáctica de equipo y genera un informe de su implementación. Esta actividad resulta muy interesante debido a que el equipo de profesores ha producido conjuntamente la guía didáctica, pero la implementación es individual y en condiciones distintas. Esto permite confrontar "lo esperado" o previsto con tres experimen-

Actividad 3. Implementación del problema "Previsión de ventas para Desigual" y su análisis

Foro de consulta

Pautas para la actividad 3

Serrano, Bosch y Gascón (2013)

Actividad 3. Informe de experimentación (o diario)

Figura 8. Actividad 3 en el espacio de la UA en la plataforma Moodle

\section{Informe de Manuel.}

El informe de Manuel indica que la actividad se implementó con un grupo de cuatro estudiantes voluntarios. En la tarea 1, eligieron una función afín (Figura 9), justificando su elección así: "esta recta tocaba el punto $\mathrm{F}$ en la gráfica y lo consideraron como el punto central, a partir del cual los demás puntos quedaban más cercanos a la recta”.

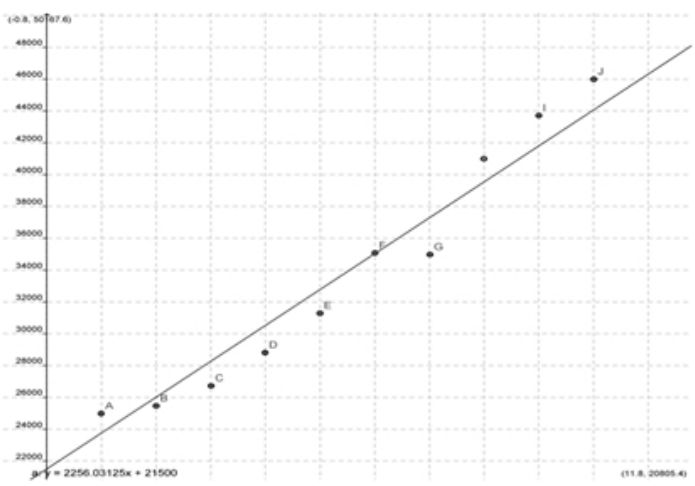

Figura 9. Modelo lineal elegido por el grupo de estudiantes taciones y realidades escolares diferentes. Para ilustrar la manera en que los profesores reportaron la experimentación, se analizan elementos de dos informes del equipo A. El de Manuel que corresponde a una experimentación con un grupo de alumnos voluntarios y el de Salvador que corresponde a una implementación en las condiciones normales del aula.

En la tarea 2, propusieron una parábola y justificaron su elección así: "porque es la más cercana a los demás puntos y el obtener una función que pasara por los demás puntos era demasiado difícil y les llevaría más tiempo, así que acordaron tomar ese modelo como el mejor".

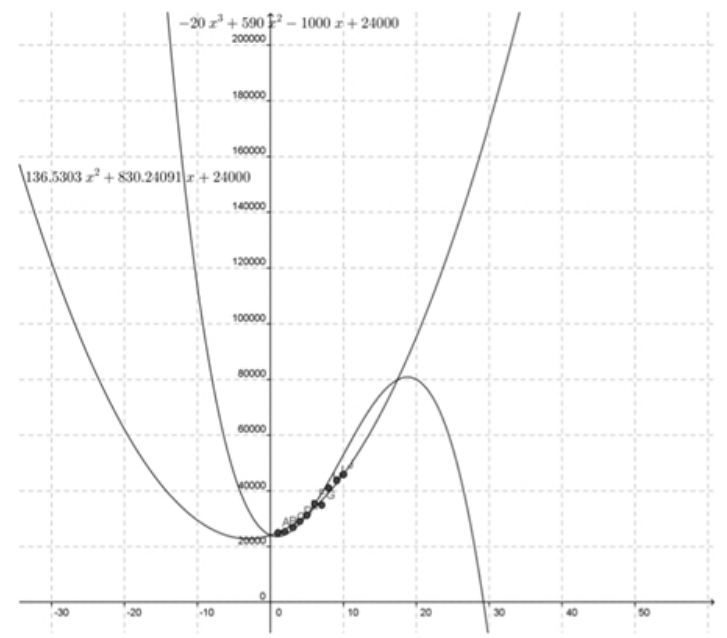

Figura 10. Modelo cuadrático vs. modelo cúbico

En la tarea 3 eligieron la función cúbica como la más adecuada indicando: "Sí, ya que los otros modelos van perdiendo credibilidad mientras más alta es la función". Al ser cuestionados por el profesor Manuel, uno de los estudiantes indicó que: "no creían que la empresa ganara siempre más y más ingresos, sino que debido a la economía lo más seguro era que quebrara en poco tiempo".

En cuanto al trabajo en equipo, Manuel indica que se discutió poco y que uno de 
los estudiantes, el que tenía mayor manejo de GeoGebra, fue quien lideró el trabajo. Asimismo, señala que la validación de los modelos fue hecha tratando "de acercarse lo más posible a todos los puntos", tomando uno o dos en particular para definir el modelo. Señaló que los conocimientos previos sobre funciones son imprescindibles y que los datos de la empresa podrían ser más amplios.

En definitiva, Manuel no consigue que los alumnos se planteen realmente el problema del ajuste, ya que éstos se contentan con argumentos débiles relacionados con criterios seudo-económicos. Es decir, criterios basados en la economía sin que haya una investigación en esta área que sustente sus

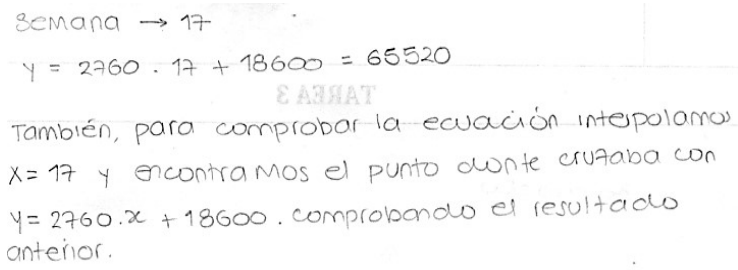

Figura 11. Previsión afín del equipo 1

En la tarea 2 que propone encontrar el mejor ajuste cuadrático, el equipo 1 mantiene el criterio anterior, misma cantidad de puntos por dentro que por fuera. El equipo 2, en cambio, elige otra parábola sin explicitar los motivos de su elección.

La representación de la función cúbica, tarea 3, genera respuestas contrarias: el equipo 1 asegura que éste es el mejor ajuste, considerando los períodos de crecimiento y argumentaciones. Sin duda el carácter voluntario de la actividad podría explicar que los alumnos se involucren poco.

\section{Informe de Salvador.}

Salvador implementó la actividad en una de sus clases del bachillerato internacional conformada por ocho estudiantes, divididos en dos equipos de cuatro. El equipo 1 eligió una recta considerando "la cantidad de puntos que quedaban "por debajo" y "por encima" de dicha recta". El equipo 2 eligió la recta que pasa por el primero y el último punto. Para hacer la previsión de la semana 17 , los dos equipos sustituyen el valor en las funciones elegidas sin mayor dificultad (Figuras 11 y 12).



Figura 12. Previsión afín del equipo 2

de pérdida de cualquier empresa: mientras que el equipo 2 asegura que no es coherente con el auge y crecimiento de la empresa, ya que conduce a la quiebra a largo plazo. Finalmente, el equipo 2 elige un ajuste cuadrático mientras que el equipo 1 elige el cúbico. Sus justificaciones se mantienen en el primer nivel de análisis, distancias menores entres los puntos y el ajuste, y en una interpretación apoyada en las representaciones gráficas (Figuras 13 y 14).

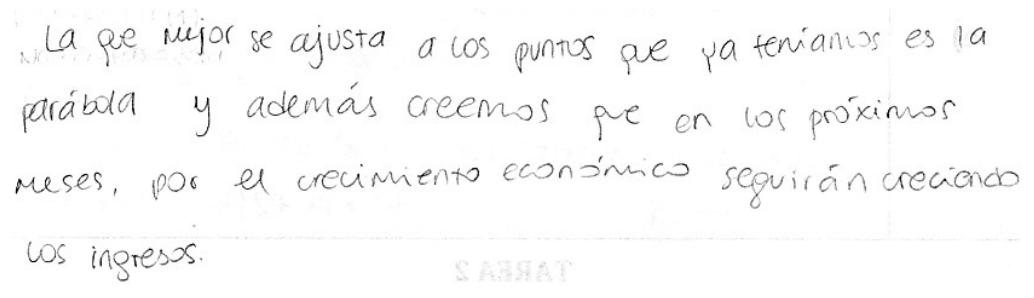

Figura 13. Previsión final del equipo 2 


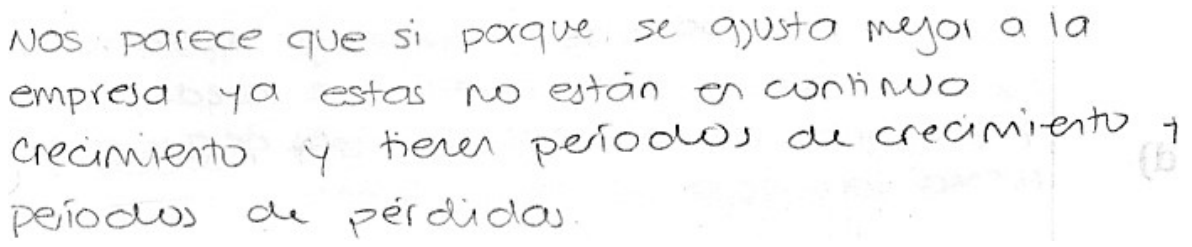

Figura 14. Previsión final del equipo 1

Salvador indica que guiar a los estudiantes a justificar matemáticamente la elección de los modelos constituye una de las dificultades más grandes en la implementación de la actividad. Para superarla, propuso calcular diferencias entre los valores dados por la empresa y los de la previsión, pero los alumnos se mantuvieron en una discusión económica; Salvador permite el debate entre los equipos y el criterio del equipo 2 termina imponiéndose.

Estos dos informes muestran que la propuesta de actividad diseñada por los profesores requiere ser modificada considerando estas experimentaciones. La confrontación de un análisis a priori y a posteriori de la experimentación es un elemento metodológico fundamental en el análisis de la implementación de tareas diseñadas o adaptadas en la Didáctica de las Matemáticas (Barquero y Bosch, 2015), y se introdujo en la Actividad 4 de la UA.

\section{Actividad 4.}

\section{Análisis conjunto y revisión final de la guía didáctica.}

La Actividad 4 tiene por objetivo generar un análisis global de la actividad de previsión de ventas. Para ello, primero se comparten en un foro por equipo los informes de las experimentaciones individuales y se analizan. Posteriormente, se revisa y modifica la guía didáctica de equipo producida en la Actividad 2, haciendo explícitos un análisis matemático y didáctico de la actividad. Para realizar la nueva guía se entrega un documento teórico producido por los forma- dores, en el que se presentan herramientas de análisis didáctico para la descripción del trabajo matemático, para gestionar y regular las actividades abiertas de modelización e investigación en comparación con las más tradicionales de transmisión de conocimiento: profesor guía del estudio y estudiante con mayor responsabilidad y autonomía, etc. Por último, se pide un apartado especial para comparar las condiciones previamente establecidas para realizar las experimentaciones y las restricciones (individuales o compartidas) que han surgido durante su implementación en las interacciones con los formadores (videos, retroalimentaciones, $\mathrm{y}$ foros). Se pone un énfasis especial en la identificación de restricciones institucionales que los profesores no habían previsto y que surgieron inevitablemente durante la experimentación.

\section{Análisis desarrollado por el equipo $C$.}

El equipo $\mathrm{C}$ presenta el análisis del REI "Previsión de ventas para Desigual" en términos de cuestiones y respuestas. Estas acciones están conformes con el documento guía para el desarrollo de esta cuarta actividad, en el que se ilustra que un REI puede ser analizado mediante una cuestión inicial, cuestiones y respuestas derivadas que generan nuevas preguntas y respuestas. Para ilustrar el análisis realizado por el equipo $\mathrm{C}$ se presentan las primeras cuestiones y respuestas, que ellos plantean:

$\mathrm{C}_{0}$ : ¿Cómo predecir ingresos futuros de la tienda de la calle Arcs 10 y ventas de camisetas one-print en la tien- 
da de Passeig de Gràcia 47, ambas de la empresa Desigual, con los datos de las tablas 7 y 8 de ("Procesos de Institucionalización", 2014)?

$\mathrm{R}_{0}$ : Dar un valor al azar parecido al que tienen las tablas, al tanteo. Esto es difícil de argumentar por lo que no se considera como una buena previsión.

$\mathrm{C}_{1}$ : ¿Cómo argumentar matemáticamente la respuesta anterior?

$\mathrm{R}_{1}$ : El tanteo no tiene ninguna argumentación, pero podemos usar un software (Excel o Minitab) para buscar una solución que investigaremos posteriormente con el fin de proporcionar buenos argumentos matemáticos. Nos enfocaremos en Minitab porque es el software oficial del curso.

$\mathrm{C}_{2}:$ ¿Qué soluciones se pueden visualizar con el uso de Minitab?

Este análisis matemático del REI finaliza reconociendo el carácter abierto del REI, en el que no es posible a priori considerar todo lo que los estudiantes pueden realizar: "El análisis matemático a priori es acorde con el contexto de los estudiantes, aunque debemos estar dispuestos a encontrar respuestas diferentes al problema planteado y a guiar la búsqueda de nuevos modelos." [Equipo C, reporte actividad 4].

En el análisis didáctico se muestra la actividad esperada del profesor y de los estudiantes, en las que parece convivir elementos de la pedagogía tradicional y de la pedagogía del cuestionamiento del mundo asociada a los REI. De acuerdo con la pedagogía tradicional, las actividades didácticas se proponen para enseñar contenidos. Los profesores lo explicitan de la siguiente manera: "Los alumnos podrán obtener como conocimientos nuevos con esta actividad: el concepto de pronóstico a corto y largo plazo, el uso de gráficas de dispersión, series del tiempo, media móvil, ajustes de pronósticos de función cuadrática, exponencial, función logística; dependiendo del grado de profundidad que logren los alumnos" [Equipo C, reporte actividad 4]. Pero, también reconocen que hay elementos más allá de los contenidos, como es la forma de validar los resultados obtenidos: "Los alumnos deben encontrar criterios para validar sus respuestas como el crecimiento indefinido en la cuadrática o el estacionamiento en la media móvil, y el mejor ajuste del pronóstico para verificar un valor conocido, por ejemplo. No necesariamente se requiere de la validación del profesor". [Equipo C, reporte actividad 4].

La implementación realizada por este equipo fue con estudiantes voluntarios y eso dificultó su compromiso en el desarrollo del REI. Además, los profesores reconocieron que el uso de la cámara incomodó a los estudiantes y que les hubiera gustado tener herramientas metodológicas más propicias para obtener más datos sobre la actividad que desarrollaron. Más allá de estas complicaciones, reconocieron que la guía didáctica puede innovarse a partir de esta primera experimentación, sin dejar de reconocer que implementar un REI en instituciones "típicas" o "tradicionales" requiere un trabajo continuo de rediseño didáctico del REI, de análisis de las experimentaciones, pero sobre todo de la instauración de un nuevo paradigma didáctico que deberá superar las restricciones institucionales actuales: "Quizá la cuestión más ambiciosa viene planteada directamente por Chevallard (2004). ¿Cómo hacer para adoptar un nuevo paradigma en el que no sean más importantes los saberes a enseñar, sino que el aprendizaje sea guiado por cuestiones a las que hay que dar respuestas? Debido a que estamos inmersos en instituciones con planes tradicionalistas, el implementar este tipo de actividades dentro del currículo, atrae muchos problemas algunos de los cuales son planteados por Chevallard. Si se aplica una actividad de éstas en el curso de Cálculo y lo que aprenden los estudiantes 
es Estadística, ¿cómo decir: “aprobaron su curso de Cálculo porque aprendieron Estadística"?" [Equipo C, reporte actividad 4].

\section{Resultados y reflexiones sobre el dise- ño de la UA y su implementación}

La investigación que se ha presentado tiene un carácter exploratorio evidente $\mathrm{y}$, en coherencia con la metodología clínica adoptada, ha tenido continuidad en las nuevas ediciones de la UA implementadas y en las programadas para los próximos años. La metodología de los REI-FP propuesta por Ruiz-Olarría (2015) ha sido adaptada al caso de la formación online en el marco institucional de las UA del CICATA, particularmente, la producción de la guía didáctica resulta un elemento clave para que los profesores generen distintos análisis matemáticos y didácticos del REI vivido, rediseñado e implementado. Sin embargo, es evidente que cada nueva edición de la UA y el análisis clínico subsiguiente pondrán de manifiesto limitaciones y debilidades de los dispositivos introducidos, (por ejemplo, el momento de presentar las herramientas teóricas y la forma de hacerlo) conduciendo en cada caso a nuevos desarrollos de éstos. De todos modos, los resultados obtenidos hasta la fecha permiten destacar algunas conclusiones provisionales en relación con los dos objetivos de esta investigación:

Analizar los efectos de la implementación de una UA y la forma en que ésta posibilita a los profesores analizar, adaptar, validar y desarrollar una propuesta didáctica sobre la enseñanza de la modelización matemática.

Observar en qué medida los profesores logran, durante la UA y gracias a las herramientas propuestas en ella, identificar las principales restricciones institucionales que dificultan la integración de la modelización matemática como actividad normalizada en el aula.
En particular, se considera necesario tomar en cuenta la potencialidad de las herramientas multimedia de la web 2.0 para lograr mayor interacción entre los formadores y los profesores a lo largo del proceso de formación, poniendo más énfasis en las modalidades de video y redes sociales. Sin embargo, estas modificaciones requieren un proceso de aprendizaje tanto por parte de los profesores como de los formadores que sin duda no tiene una solución única ni óptima. Sin embargo, la pericia en el manejo de estas herramientas resulta necesaria para que los profesores puedan actuar como "actores de cambio", tal como menciona Costa (2009).

Respecto a la manera en que los profesores han mostrado capacidades para analizar, adaptar, validar y desarrollar una propuesta didáctica sobre la enseñanza de la modelización matemática, se considera que se debe a que en el diseño de la UA se tiene en cuenta tanto sus conocimientos profesionales como la transposición de los elementos teóricos que sustentan el mismo diseño. Los profesores que han participado en esta UA tienen experiencia docente heterogénea, muchos o pocos años de servicio, enseñanza en diferentes niveles educativos y en diversas instituciones. Por tanto, los saberes docentes son considerados existentes y se solicita que sean reflejados en las Actividades 2, 3 y 4. Sin embargo, dichos conocimientos están fuertemente moldeados por la pedagogía dominante y es posible debatir su existencia gracias a la experiencia del REI vivido (Actividad 1), ya que se implementa bajo otra pedagogía distinta: la del Cuestionamiento del Mundo (Chevallard, 2013).

En cuanto al difícil tema de las restricciones institucionales que dificultan la integración de la modelización matemática como actividad normalizada en el aula, se considera que la UA propuesta constituye una herramienta valiosa para permitir a los 
profesores avanzar en este aspecto. En efecto, la estrategia propuesta en la Actividad 2 de diseño espontáneo de una tarea docente sobre modelización matemática evidencia las principales restricciones institucionales en las que evolucionan los profesores con normalidad: "cierre" de la actividad de modelización a una secuencia de preguntas cortas; imposición de ciertos modelos matemáticos previamente identificados por el profesor; un uso muy dirigido de las TIC; predominio del contenido matemático curricular frente al problema por resolver. De todas formas, como se observa en la Actividad 4 basada en el análisis de la experimentación, los profesores son capaces de identificar a posteriori muchas de estas restricciones, aun cuando algunos de ellos no las hayan sabido anticipar. Algunas de las respuestas al cuestionario de evaluación final fueron:

R: Un planteamiento global y unificador del curso que nos permitió, desde los diversos roles desde los que jugamos, tener una mirada crítica de nuestro trabajo como docentes y una re-lectura del rol de estudiante.

J: Es muy interesante observar como las instituciones marcan en cierta forma el actuar del docente.

P: Me parece que el curso está lleno de matices que cambian nuestra expectativa al jugar diferentes roles, observar cómo la institución donde laboro me ha influenciado en las cuestiones administrativas (realizar guía didáctica), reflexionar sobre cómo mi formación conductista aún me "persigue" en mi acción docente, contribuir al trabajo colaborati- vo donde el intercambio de experiencias vuelve más enriquecedor el producto final, proporciona una visión muy optimista al respecto.

Finalmente, volviendo al problema de las posibilidades y límites de la formación online de los profesores de matemáticas teniendo en cuenta los principios que plantea Scott (2010), se puede afirmar que la UA implementada se inscribe en la dirección propuesta: toma como punto de partida un problema abierto de la profesión docente; se basa en un trabajo colaborativo guiado por un grupo de formadores expertos en didáctica de las matemáticas; permite superar la división "teoría-práctica" mencionada por esta autora, poniendo al alcance de los profesores herramientas innovadoras; facilita la experimentación de nuevas estrategias docentes que incorporan el uso de las TIC. De esta forma, se promueve la adaptación crítica de recursos didácticos, el compartir, analizar y evaluar entre pares las actividades implementadas en el aula, ayudados por la activación de las herramientas teóricas aprendidas en la UA.

Esta investigación se basa de momento en cuatro experimentaciones singulares, implementando el mismo REI, que no permiten mucha generalización. Pero aportan optimismo en las inmensas posibilidades que ofrece la formación online y a distancia del profesorado, alcanzando profesionales docentes de comunidades educativas muy alejadas y creando redes de cooperación que todavía no sabemos cómo explotar mejor.

\section{Agradecimientos}

Esta investigación se ha desarrollado en el marco de los proyectos $\mathrm{I}+\mathrm{D}+\mathrm{i}$ "Propuestas para una enseñanza basada en el paradigma del cuestionamiento del mundo" (Q-mundo): RTI2018-101153-A-C22 y RTI2018-101153-B-C21 del Programa Estatal de I+D+i Orientada a los Retos de la Sociedad. 


\section{REFERENCIAS BIBLIOGRÁFICAS}

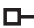

Artigue, M. (2016). Soutenir le développement professionnel des enseignants: un défi majeur à relever. Cuadernos de Investigación y Formación en Educación Matemática, 11(15), 35-56.

Barberá, J.P., y Fuentes, M. (2012). Aprender inteligencia emocional mediante formación online ¿es posible? Revista Didáctica, Innovación y Multimedia, 24 http://dim.pangea.org/revista24.htm

Barquero, B., \& Bosch, M. (2015). Didactic Engineering as a Research Methodology: From Fundamental Situations to Study and Research Paths. In A. Watson \& M. Ohtani (Eds.), Task Design in Mathematics Education- ICMI Study 22 (pp. 249-273). New ICMI Study Series. Cham: Springer. doi:10.1007/978-3-319-09629-2_8

Barquero, B., Florensa, I., \& Ruiz-Olarría, A. (2019). The education of school and university teachers within the paradigm of questioning the world. In M. Bosch, Y. Chevallard, F. J. García y J. Monaghan (Eds.), Working with the anthropological theory of the didactic: A comprehensive casebook (pp. 189-212). New York, USA: Routledge

Barquero, B., Bosch, M., \& Romo, A. (2018). Mathematical modelling in teacher education: dealing with institutional constraints. ZDM - Mathematics Education, 50(1-2), 31-43. doi:10.1007/ s11858-017-0907-z

Borba, M. C., \& Zulatto, R. B. A. (2006). Different media, different types of collective work in online continuing teacher education: Would you pass the pen, please? In Proceedings of the $30^{\text {th }}$ conference of the international group for the psychology of mathematics education (pp. 201-208). Prague: PME

Bosch, M., \& Gascón, J. (2009). Aportaciones de la Teoría Antropológica de lo Didáctico a la Formación del Profesorado de Secundaria. In M. L. González, M. T. González \& J. Murillo (Eds.) Investigación en Educación Matemática XIII (pp. 89-113). Santander, España: SEIEM.

Chevallard, Y. (2013). Enseñar matemáticas en la sociedad de mañana: Alegato a favor de un contraparadigma emergente. Redimat - Journal of Research in Mathematics Education, 2(2), 161-182. doi:10.4471/redimat.2013.26

Cirade, G. (2006). Devenir professeur de mathématiques. Entre problèmes de la profession et formation à l'IUFM. (Tesis de doctorado). Université de Provence, Francia.

Castañeda, L., \& Adell, J. (2011). El desarrollo profesional de los docentes en entornos personales de aprendizaje (PLE). En Rogi Vila, R. y Laneve, C. (Eds.) La práctica educativa en la Sociedad de la Información: Innovación a través de la investigación (pp. 83-95). Alcoy: Marfil.

Costa, C. (2009). Teachers professional development through Web 2.0 environments. Best practices for the knowledge society. Knowledge, learning, development and technology for all. Communications in Computer and Information Science, 49, part 1, 26-32. 
Galleguillos, J., \& Borba, M. (2017). Expansive movements in the development of mathematical modeling: analysis from an Activity Theory perspective. ZDM - Mathematics Education, 44(6), 717-731. doi:10.1007/s11858-017-0903-3

Garuti, R. (2004). From research in mathematics education to teachers' training through Internet. En M.A. Mariotti (Ed.), Proceedings of the Third Conference of the European Society for Research in Mathematics Education (pp. 1-9). Italy: University of Pisa.

Gueudet, G., Sacristán, A.I., Soury-Lavergne, S., \& Trouche, L. (2012). Online paths in mathematics teacher training: new resources and new skills for teacher educators. ZDM-Mathematics Education, 44(6), 717-731.

Goldsmith L. T., Doerr, H., M., \& Lewis, C. (2014). Mathematics teachers' learning: a conceptual framework and synthesis of research. Journal of Mathematics Teacher Education 17(1), 5-36. doi:10.1007/s10857-013-9245-4

Gómez-Blancarte, A., Romo-Vázquez, A., Miranda, I., Aguilar, M.S., Castañeda, A., \& Lezama, J. (2019). An online learning community for the professional development of mathematics teachers in Mexico. Interciencia, 44(4), 247-252.

Llinares, S., \& Valls, J. (2010). Prospective primary teachers' learning from on-line discussions in a virtual video-based environment. Journal of Mathematics Teacher Education, 13(2), 177-196. doi:10.1007/s10857-009-9133-0

McGraw, R., Lynch, K., Koc, Y., Budak, A., \& Brown, C.A. (2007). The multimedia case as a tool for professional development: an analysis of online and face-to-face interaction among mathematics pre-service teachers, in-service teachers, mathematicians, and mathematics teacher educators. Journal of Mathematics Teacher Education, 10(2), 95-121. doi:10.1007/s10857-007-9030-3

Orey, D.C., \& Rosa, M. (2018). Developing a mathematical modelling course in a virtual learning environment. ZDM - Mathematics Education 50(1-2), 173-185. doi:10.1007/s11858-018-0930-8

Parra-Zapata, M. M., Rendón-Mesa, P. A., Ocampo-Arenas, M. C., Sánchez-Cardona. J., Molina-Toro, J.F., y Villa-Ochoa, J. A. (2018). Participación de profesores en un ambiente de formación online. Un estudio en modelación matemática. Educación Matemática, 30(1), 185-212. doi:10.24844/EM3001.07

Pedinielly, J.L., y Fernandez, L. (2015). L'observation clinique et l'étude de cas (3e). París: Armand Colin.

Ponte, J.P., \& Santos, L. (2005). A distance in-service teacher education setting focused on mathematics investigations: the role of reflection and collaboration. Interactive Educational Multimedia, 11, 104-126.

Romo, A., y Covián, O.N. (2018). Desarrollo profesional de profesores de matemáticas en la modalidad en línea y a distancia. El caso de un curso para el diseño de actividades didácticas. REDIMAT - Journal of Research in Mathematics Education, 7(1), 69-92. doi:10.17583/redimat.2018.2016 
Romo-Vázquez. A., y Gómez-Blancarte, A. L. (2018). La equidad en el aprendizaje de profesores mediante la interacción entre experiencia y competencia. Revista Colombiana de Educación, 74 , 269-287.

Romo-Vázquez, A., y Hache, C. (2019). Prácticas lingüísticas en la clase de matemáticas. Una experiencia de profesionalización online para profesores de matemáticas. REDIMAT - Journal of Research in Mathematics Education, 8(2), 112-138. doi:10.4471/redimat.2019.2503

Ruiz-Olarría, A. (2015). La formación matemático-didáctica del profesorado de secundaria: De las matemáticas por enseñar a las matemáticas para la enseñanza. (Tesis de doctorado). Universidad Autónoma de Madrid, Madrid, España.

Serrano, L., Bosch, M., y Gascón, J. (2013). Recorridos de estudio e investigación en la enseñanza universitaria de ciencias económicas y empresariales. UNO: Revista de didáctica de las matemáticas, 62, 39-48.

Serrano, L. (2002). La modelización matemática en los estudios universitarios de economía y empresa: análisis ecológico y propuesta didáctica. (Tesis de doctorado). Universidad Ramon Llul, Barcelona, España.

Sierra, T. A. (2006). Lo matemático en el diseño y análisis de organizaciones didácticas. Los sistemas de numeración y la medida de magnitudes (Tesis de doctorado). Universidad Complutense de Madrid, Madrid, España.

Scott, S. (2010). The theory and practice divide in relation to teacher professional development. En J. O. Lindberg \& D. O. Angers (eds.), Online learning communities and teacher professional development: Methods for improved education delivery (pp. 20-40). Hershey, USA: IGI Global.

Winbourne, P., \& Watson, A. (1998). Participating in learning mathematics through shared local practices in classrooms. In A. Watson (Ed.), Situated cognition and the learning of mathematics (pp. 93-104). Oxford, Inglaterra: Centre for Mathematics Education Research. University of Oxford.

Wideman, H. (2010). Online teacher learning communities: A literature review. Institute for Research on Learning Technologies, York University. 